\title{
Rainbow modified-ladder approximation and degenerate pion
}

\author{
Lei Chang $\odot^{1, *}$ and Minghui Ding $\odot^{2, \dagger}$ \\ ${ }^{1}$ School of Physics, Nankai University, Tianjin 300071, China \\ ${ }^{2}$ European Centre for Theoretical Studies in Nuclear Physics and Related Areas (ECT*) \\ and Fondazione Bruno Kessler, Villa Tambosi, Strada delle Tabarelle 286, I-38123 Villazzano (TN), Italy
}

(Received 19 November 2020; accepted 10 March 2021; published 2 April 2021)

\begin{abstract}
Correlation functions can be described by the corresponding equations, viz., the gap equation for the quark propagator and the inhomogeneous Bethe-Salpeter equation for the vector dressed-fermion-Abeliangauge-boson vertex in which specific truncations have to be implemented. The general vector and axialvector Ward-Green-Takahashi identities require these correlation functions to be interconnected; in consequence of this, truncations made must be controlled consistently. It turns out that, if the rainbow approximation is assumed in the gap equation, the scattering kernel in the Bethe-Salpeter equation can adopt the ladder approximation, which is one of the most basic attempts to truncate the scattering kernel. Additionally, a modified-ladder approximation is also found to be a possible symmetry-preserving truncation scheme. As an illustration of this approximation for application, a treatment of the pion is included. The pion mass and decay constant are found to be degenerate in ladder and modified-ladder approximations, even though the Bethe-Salpeter amplitudes are with apparent distinction. The justification for the modified-ladder approximation is examined with the help of the Gell-Mann-Oakes-Renner relation.
\end{abstract}

DOI: 10.1103/PhysRevD.103.074001

\section{INTRODUCTION}

The hadron is a composite particle consist of quarks and gluons that are strongly interacting, so it cannot be described by perturbation theory. Rather, the constituent quark model and parton model have been historically linked to the instructive description of the hadron, so much so that it seems their developments are progressively paving our way to gain greater insight directly from quantum chromodynamics (QCD) [1-4]. Yet, how QCD gives rise to the physics of the hadron is a question that has not been mathematically answered. This intractable aspect of the theory is investigated by lattice QCD, which has already successfully agreed with many experiments on nonperturbative phenomena [5]. Meanwhile, a continuum field approach can serve as a complement, such as DysonSchwinger equations (DSEs) [6-8] and the functional renormalization group $[9,10]$. More precisely, the DSE, for instance, is a nonperturbative approach that can manifest main properties of QCD: dynamical chiral symmetry breaking (DCSB) and color confinement. It is yielding

\footnotetext{
*leichang@ nankai.edu.cn

†mding@ectstar.eu
}

Published by the American Physical Society under the terms of the Creative Commons Attribution 4.0 International license. Further distribution of this work must maintain attribution to the author(s) and the published article's title, journal citation, and DOI. Funded by SCOAP ${ }^{3}$. progress on hadron phenomena, in particular, that of the lightest meson - the pion. Despite being a bound state, the pion is the pseudo Nambu-Goldstone mode generated by DCSB, and this dichotomous character entails that it takes a special position among theoretical interests on the hadron $[11,12]$. The point to maintain this dichotomous character of the pion is obeying the fundamental symmetry in QCD. That in DSE is to follow some basic rules when truncating the infinite coupled equations for correlation functions $[13,14]$. Herein, the starting points are the quark gap equation and the Bethe-Salpeter equations (BSEs).

The rainbow approximation for the quark gap equation and the ladder approximation for BSE have been developed for decades $[15,16]$ and applied to a wide range of hadronic systems, including meson, baryon, and exotic states, etc. [6-8]. The underlying law governing their application in a simple and elegant way is the preserving of vector and axial-vector Ward-Green-Takahashi identities driven by gauge symmetry. They lead to peculiar relationships between truncations made for the quark gluon vertex in the gap equation and the scattering kernel in the BetheSalpeter equation. Two nonlinear equations, which connect the quark gluon vertex with the scattering kernel, can be acquired to exhibit the symmetry-driven correspondence. Starting from these two equations, one notices that if the quark gluon vertex is assumed to be a bare vertex, i.e., the rainbow approximation as it is usually called, then a straightforward expression for the scattering kernel is immediately realized to be valid, which is interpreted as 
the ladder approximation. Given the existence of this nontrivial solution, i.e., rainbow ladder approximation, for two nonlinear equations, one would be inclined to expect that other solutions may also exist.

Attempts can be made in two directions searching for other solutions. One of the directions is to go beyond both rainbow and ladder approximations, consistently truncating the quark gluon vertex in DSE and the scattering kernel in BSE [17-24]. It turns out that this direction is indeed workable in practice. Additionally, it has been proved that if the quark gluon vertex is added with corrections to the bare vertex, viz., beyond rainbow approximation, the Nambu-Goldstone theorem is manifest only if the scattering kernel is constructed consistently [25]. With the extension of the rainbow ladder approximation in this direction, one may acquire a good description of the spin-orbit splitting in the light meson [26,27], the level ordering of pseudoscalar and vector meson radial excitations [28], and the heavy-light meson mass spectrum [29]. One may acquire further potential access characterizing meson internal structures, such as distribution amplitude [30] and resonance width [31,32].

An alternative direction is to keep the rainbow approximation and instead modify the ladder approximation $[33,34]$. We seek for this possibility in this work, assuming the ladder approximation can include a multiplicative factor, which can be recognized as our ansatz for the scattering kernel. This introduced factor will be determined by the two nonlinear equations constrained from the preserving of vector and axial-vector Ward-GreenTakahashi identities. In this case, through imposing this nontrivial multiplicative factor, we find two other possible solutions for the two nonlinear equations. They are rainbow modified-ladder approximations. This procedure of expressing the scattering kernel in terms of ladder approximation with a multiplicative factor and deriving its wellconstrained form is purely mathematical, independent of the system under consideration. To explore whether the rainbow modified-ladder approximations are useful in a practical system, we include their applications on the pion, examining whether the Nambu-Goldstone theorem is manifest with modified-ladder approximations, as well as studying their impacts on the internal structure of the pion. Properties such as pion mass, Bethe-Salpeter amplitude, and decay constant are considered in comparison with those in the rainbow ladder approximation. Additionally, the reasonability for modified-ladder approximation is provided by the verification of the Gell-Mann-OakesRenner (GMOR) relation.

The remainder of this paper is organized as follows. In Sec. II, we reiterate the vector and axial-vector WardGreen-Takahashi identities, highlighting two corresponding nonlinear equations for the quark gluon vertex and the scattering kernel. Section III introduces the rainbow ladder approximation together with the rainbow modified-ladder approximation. Section IV deals with the application of rainbow modified-ladder approximation on the pion. Section V contains our results of the pion mass, BetheSalpeter amplitude, and decay constant, as well as the discussion on the GMOR relation. Finally, we summarize in Sec. VI.

\section{VECTOR AND AXIAL-VECTOR WARD-GREEN-TAKAHASHI IDENTITIES}

Quantum chromodynamics provides underlying laws governing the properties of particles, both elementary and hadronic. It requires the correlation functions to be interconnected by the Slavnov-Taylor identity, which corresponds to the Ward-Green-Takahashi identity in an Abelian gauge. We consider herein the connections between the three-point vertex of a dressed fermion to an Abelian gauge boson and the two-point function, i.e., quark propagator. These connections are expressed by the vector and axial-vector Ward-Green-Takahashi identities.

\section{A. Vector Ward-Green-Takahashi identity}

The Ward identity [35], and its generalization by Green [36] and Takahashi [37], is

$$
i P_{\mu} \Gamma_{\mu}(k ; P)=S^{-1}\left(k_{+}\right)-S^{-1}\left(k_{-}\right),
$$

which relates the three-point function, the vector dressedfermion-Abelian-gauge-boson vertex $\Gamma_{\mu}(k ; P)$ with the two-point function, the quark propagator $S(k)$. The original Ward identity, derived earlier by Ward from a study of perturbation theory, can be obtained from letting $k_{+}$ approach $k_{-}$, with $k_{+}=k \pm P / 2$, and $k$ and $P$ are, respectively, the relative and total momentum of the dressed quark and dressed antiquark.

The quark propagator in Eq. (1) satisfies the gap equation $^{1}$

$$
\begin{aligned}
S^{-1}(k)= & Z_{2}\left(i \gamma \cdot k+Z_{m} m^{\zeta}\right) \\
& +Z_{2}^{2} \int_{d q} g^{2} \mathcal{D}_{\mu \nu}(k-q) \frac{\lambda^{a}}{2} \gamma_{\mu} S(q) \frac{\lambda^{a}}{2} \Gamma_{\nu}(k, q),
\end{aligned}
$$

where $m^{\zeta}$ is the current quark mass and $\zeta$ is the renormalization scale; $Z_{2, m}$ are, respectively, quark wave function and mass renormalization constants (the term $Z_{2}^{2}$ in quark self-energy may be understood from the constraint of multiplicative renormalizability of the quark propagator as explored in Ref. [38]); $\mathcal{D}_{\mu \nu}(k-q)$ is the gluon

\footnotetext{
${ }^{1}$ We use a Euclidean metric: $\left\{\gamma_{\mu}, \gamma_{\nu}\right\}=2 \delta_{\mu \nu} ; \gamma_{\mu}^{\dagger}=\gamma_{\mu}$; $\gamma_{5}=\gamma_{4} \gamma_{1} \gamma_{2} \gamma_{3}, \quad \operatorname{tr}\left[\gamma_{4} \gamma_{\mu} \gamma_{\nu} \gamma_{\rho} \gamma_{\sigma}\right]=-4 \epsilon_{\mu \nu \rho \sigma} ; \quad \sigma_{\mu \nu}=(i / 2)\left[\gamma_{\mu}, \gamma_{\nu}\right]$; $a \cdot b=\sum_{i=1}^{4} a_{i} b_{i}$; and $P_{\mu}$ timelike $\Rightarrow P^{2}<0$. More information is available in Sec. 2.3 in Ref. [6].
} 
propagator; $\Gamma_{\nu}(k, q)$ is the quark gluon vertex; and $\lambda^{a}$ are the Hermitian Gell-Mann matrices.

As outlined in the preceding introduction, we seek for a possible modified-ladder approximation at the same time keeping rainbow approximation. In the following discussion, we will implement the rainbow approximation in the gap equation, and, in this case, the quark gluon vertex in Eq. (2) is the bare vertex as

$$
\Gamma_{\nu}(k, q)=\gamma_{\nu}
$$

The three-point function with a specified $J^{P}$ quantum number satisfies the general inhomogeneous BetheSalpeter equation

$$
\begin{aligned}
{\left[\Gamma_{J^{P}}(k ; P)\right]_{\alpha \beta}=} & Z_{J^{P}}\left[\gamma_{J^{P}}\right]_{\alpha \beta}+Z_{2}^{2} \int_{d q}[\mathcal{K}(k, q, P)]_{\alpha \alpha^{\prime} ; \beta^{\prime} \beta} \\
& \times\left[S\left(q_{+}\right) \Gamma_{J^{P}}(q, P) S\left(q_{-}\right)\right]_{\alpha^{\prime} \beta^{\prime}}
\end{aligned}
$$

with $\mathcal{K}(k, q, P)$ the quark-antiquark scattering kernel. $\gamma_{J^{P}}=\mathbf{1}, \gamma_{5}, \gamma_{\mu}, \gamma_{5} \gamma_{\mu}$, etc., is the inhomogeneous driving term corresponding to the $J^{P}$ quantum number, and $Z_{J^{P}}$ is the related renormalization constant. The vertex that appeared in Eq. (1) is the vector dressed-fermion-Abeliangauge-boson vertex with $\gamma_{J^{P}}=\gamma_{\mu}$, and $Z_{J^{P}}=Z_{2}$. With the specification of quark propagator $S(k)$ and vector vertex $\Gamma_{\mu}(k ; P)$ in Eq. (1), as well as the application of the rainbow approximation, the only quantity that is unknown, $\mathcal{K}(k, q, P)$, can be well settled.

Inserting the quark propagator in Eq. (2) and the vector vertex in Eq. (4) into the vector Ward-Green-Takahashi identity expressing their connection in Eq. (1), one will have

$$
\begin{aligned}
& \int_{d q} \mathcal{K}(k, q, P)_{\alpha \alpha^{\prime} ; \beta^{\prime} \beta}\left[S\left(q_{+}\right)-S\left(q_{-}\right)\right]_{\alpha^{\prime} \beta^{\prime}} \\
& =-\int_{d q} g^{2} \mathcal{D}_{\mu \nu}(k-q) \frac{\lambda^{a}}{2} \gamma_{\mu}\left[S\left(q_{+}\right)-S\left(q_{-}\right)\right] \frac{\lambda^{a}}{2} \gamma_{\nu} .
\end{aligned}
$$

This is a relation that scattering kernel $\mathcal{K}(k, q, P)$ must preserve in the rainbow approximation.

\section{B. Axial-vector Ward-Green-Takahashi identity}

The other generalized Ward identity is

$$
P_{\mu} \Gamma_{5 \mu}(k ; P)=S^{-1}\left(k_{+}\right) i \gamma_{5}+i \gamma_{5} S^{-1}\left(k_{-}\right)-2 i m^{\zeta} \Gamma_{5}(k ; P),
$$

where $\Gamma_{5 \mu}(k ; P)$ is the axial-vector vertex, and it satisfies the inhomogeneous axial-vector Bethe-Salpeter equation in Eq. (4) with $\gamma_{J^{P}}=\gamma_{5} \gamma_{\mu}$ and $Z_{J^{P}}=Z_{2} ; \Gamma_{5}(k ; P)$ is the pseudoscalar vertex, and it satisfies the inhomogeneous pseudoscalar Bethe-Salpeter equation in Eq. (4) with $\gamma_{J^{P}}=\gamma_{5}$ and $Z_{J^{P}}=Z_{4}$.

Inserting the quark propagator in Eq. (2) and the axialvector and pseudoscalar vertices in Eq. (4) into the axialvector Ward-Green-Takahashi identity in Eq. (6), one will have

$$
\begin{aligned}
& \int_{d q} \mathcal{K}(k, q, P)_{\alpha \alpha^{\prime} ; \beta^{\prime} \beta}\left[S\left(q_{+}\right) \gamma_{5}+\gamma_{5} S\left(q_{-}\right)\right]_{\alpha^{\prime} \beta^{\prime}} \\
& =-\int_{d q} g^{2} \mathcal{D}_{\mu \nu}(k-q) \frac{\lambda^{a}}{2} \gamma_{\mu}\left[S\left(q_{+}\right) \gamma_{5}+\gamma_{5} S\left(q_{-}\right)\right] \frac{\lambda^{a}}{2} \gamma_{\nu} .
\end{aligned}
$$

This is a second relation that scattering kernel $\mathcal{K}(k, q, P)$ must preserve in the rainbow approximation.

In this way, we see that the use of vector and axialvector Ward-Green-Takahashi identities in the construction of two general relations-Eqs. (5) and (7) —for the scattering kernel in the rainbow approximation has been motivated by the requirement of gauge symmetry and chiral symmetry. These two constructed relations in the case of rainbow approximation are consistent with those equations derived in a more general case $[22,23,28]$. Notably, the procedure of constructing the scattering kernel is purely mathematical, independent of the system under consideration.

\section{QUARK-ANTIQUARK SCATTERING KERNEL}

With the recognition that mathematical formulation associated with gauge and chiral symmetries in Eqs. (5) and (7) must be preserved for the scattering kernel, we shall begin to solve these two equations, and the resulting scattering kernel will automatically keep corresponding symmetries.

\section{A. Ladder approximation}

There is one apparent practical solution, which is

$$
[\mathcal{K}(k, q, P)]_{\alpha \alpha^{\prime} ; \beta^{\prime} \beta}^{\mathrm{RL}}=-g^{2} \mathcal{D}_{\mu \nu}(k-q)\left[\frac{\lambda^{a}}{2} \gamma_{\mu}\right]_{\alpha \alpha^{\prime}} \otimes\left[\frac{\lambda^{a}}{2} \gamma_{\nu}\right]_{\beta^{\prime} \beta} .
$$

It is known as the rainbow ladder (RL) approximation. The ladder approximation has been one of the most successful attempts to truncate Dyson-Schwinger equations, which is still meaningful in plenty of recent studies [39-47]. From the mathematical viewpoint, it is recognized as the leadingorder truncation scheme, and any correction to it can provide us further assistance in our better understanding of the truncations. 


\section{B. Modified-ladder approximation}

If one solution of a nonlinear system of equations exists, it is often possible to find several solutions, and we realize this is the case herein. We now assume that the scattering kernel in Eq. (8) has an extension as

$$
\begin{aligned}
{[\mathcal{K}(k, q, P)]_{\alpha \alpha^{\prime} ; \beta^{\prime} \beta}^{\mathrm{RML}}=} & -g^{2} \mathcal{D}_{\mu \nu}(k-q)\left[\frac{\lambda^{a}}{2} \gamma_{\mu} \Lambda_{\beta}\right]_{\alpha \alpha^{\prime}} \\
& \otimes\left[\Lambda_{\beta} \frac{\lambda^{a}}{2} \gamma_{\nu}\right]_{\beta^{\prime} \beta},
\end{aligned}
$$

where $\Lambda_{\beta}$ is a Dirac structure function. A graphic representation of Eq. (9) is given in Fig. 1. Thus, the scattering kernel is considered to be the ladder approximation with a multiplicative factor. We have to admit that this is an ansatz, and whether it is reliable remains to be justified by whether we can find a nontrivial expression for $\Lambda_{\beta}$. One apparent expression for $\Lambda_{\beta}$ is the identity matrix $\mathbf{1}$, and the associated scattering kernel is the ladder approximation. We then go further to look for other possibilities. The resulting $\Lambda_{\beta}$ must be distinguished from 1 so that modified-ladder approximation is distinguished from ladder approximation.

The procedure finding nontrivial $\Lambda_{\beta}$ is mathematical. In general, $\Lambda_{\beta}$ can be of all Dirac matrices $\left\{\gamma_{5}, \frac{1}{2} \gamma_{\beta}, \frac{i \gamma_{5}}{2} \gamma_{\beta}\right.$, $\left.\frac{i}{2 \sqrt{3}} \sigma_{\beta \alpha}, \frac{i \gamma_{5}}{2 \sqrt{3}} \sigma_{\beta \alpha}\right\}$ [48]; however, practical calculation yields only the two possible following forms:

$\Lambda_{\beta}^{+}=\frac{i}{\sqrt{3}} \frac{\gamma_{5} \sigma_{\beta \alpha}\left(i q_{+}^{\alpha} \sigma_{v}\left(q_{+}^{2}\right)-i q_{-}^{\alpha} \sigma_{v}\left(q_{-}^{2}\right)\right)}{\sqrt{q_{+}^{2} \sigma_{v}^{2}\left(q_{+}^{2}\right)+q_{-}^{2} \sigma_{v}^{2}\left(q_{-}^{2}\right)-2 q_{+} \cdot q_{-} \sigma_{v}\left(q_{+}^{2}\right) \sigma_{v}\left(q_{-}^{2}\right)}}$,

$\Lambda^{-}=\frac{1 \gamma_{5} \sigma_{\beta \alpha}\left(i q_{+}^{\alpha} q_{-}^{\beta}-i q_{-}^{\alpha} q_{+}^{\beta}\right)}{2}$

where $\sigma_{\mu \nu}=\frac{i}{2}\left(\gamma_{\mu} \gamma_{\nu}-\gamma_{\nu} \gamma_{\mu}\right)$ and $q_{ \pm}=q \pm P / 2 ; \sigma_{v}\left(q^{2}\right)$ is the vector part of quark propagator $S(q)=$ $-i \gamma \cdot q \sigma_{v}\left(q^{2}\right)+\sigma_{s}\left(q^{2}\right)$.
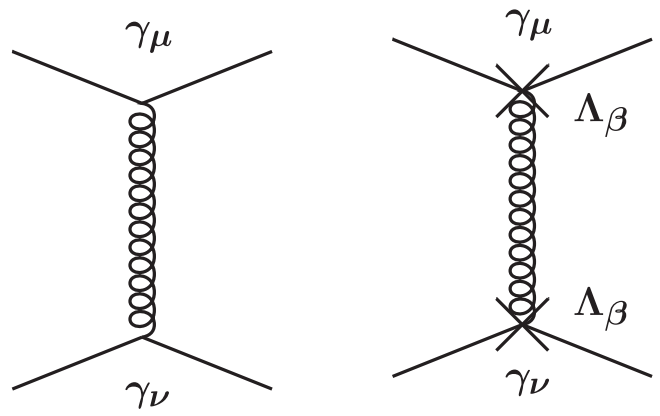

FIG. 1. Left: scattering kernel with ladder approximation in Eq. (8). Right: scattering kernel with modified-ladder approximation in Eq. (9). The internal solid lines represent dressed gluon propagators. The cross indicates to multiply a $\Lambda_{\beta}$ where it is marked.
The expressions in Eq. (10) show very clearly the difference between ladder approximation and modifiedladder approximation. In the case of ladder approximation, there is no momentum dependence in scattering kernel $\mathcal{K}(k, q, P)$ expect for the one that appears in the gluon propagator. However, there is momentum dependence in modified-ladder approximation, described by quark momentum $q_{ \pm}$and/or the vector part of quark propagator $\sigma_{v}\left(q^{2}\right)$. In consequence of this, the modification on the scattering kernel to some extent can be considered as the rearrangement of the quark momentum within the hadron.

Additionally, given the importance of the examination of whether the Nambu-Goldstone theorem is manifest in modified-ladder approximation, we specify the formulation of the expression $\Lambda_{\beta}^{+}$in Eq. (10) in the chiral limit. If a massless pion exists, then $P=0$ and $\Lambda_{\beta}^{+}=\frac{i \gamma_{5}}{\sqrt{3}} \sigma_{\beta \alpha} n_{\alpha}$, with $n=(0,0,0, i)$ the unit vector in the direction of $P$.

\section{APPLICATION: PION}

In the preceding section, we have found two mathematical expressions for rainbow modified-ladder (RML) approximation, and, in order to justify their rationality, we shall now consider their application on the lightest hadron system, the pion. The study on the pion is an apparent direction, since it is possible to consider whether the Nambu-Goldstone theorem is manifest with RML approximations, which is the basic criteria that one must meet when developing new scattering kernels [25]. The analysis on the pion mass, decay constant, and BetheSalpeter amplitude should be quite interesting affairs. It is known that the pion can be described by the homogeneous Bethe-Salpeter equation

$$
\begin{aligned}
& \lambda\left(P^{2}\right) S^{-1}\left(k_{+}\right)[\chi(k ; P)]_{\alpha \beta} S^{-1}\left(k_{-}\right) \\
& \quad=Z_{2}^{2} \int_{d q}[\mathcal{K}(k, q, P)]_{\alpha \alpha^{\prime} ; \beta^{\prime} \beta}[\chi(q, P)]_{\alpha^{\prime} \beta^{\prime}},
\end{aligned}
$$

with $\lambda\left(P^{2}\right)$ the eigenvalue of the kernel, which is equal to one when the pion is on shell: $\lambda\left(P^{2}=-M_{\pi_{i}}^{2}\right)=1$. The corresponding eigenvector of Eq. (11) is pion BetheSalpeter wave function $\chi(k, P)$. When solving this equation, we take the pion Bethe-Salpeter wave function as

$$
\chi(k ; P)=\sum_{i=1}^{4} \tau_{i}(k ; P) f_{i}(k ; P),
$$

where $f_{i}(k, P)$ is the scalar function characterizing pion internal wave function dependence on the relative and total momentum of the dressed quark and dressed antiquark. $\tau_{i}$ is the complete set of the Dirac bases for the pseudoscalar meson Bethe-Salpeter wave function [11]: 


$$
\begin{aligned}
& \tau_{1}=i \gamma_{5}, \quad \tau_{2}=\gamma_{5} \gamma \cdot P, \\
& \tau_{3}=\gamma_{5} P \cdot k \gamma \cdot k, \quad \tau_{4}=\gamma_{5} \sigma_{\mu \nu} k_{\mu} P_{\nu} .
\end{aligned}
$$

By adapting this set of Dirac bases, we find special properties for the pion Bethe-Salpeter equation with RML approximations. For instance, if multiplying the Lorentz structure of the right-hand side of Eq. (11) in the case of $\Lambda_{\beta}^{+}$ by $\tau_{i}$, which is the common procedure when solving Eq. (11), one may immediately notice

$$
\begin{array}{ll}
\operatorname{tr}\left[\tau_{i} \Lambda_{\beta}^{+} \tau_{j} \Lambda_{\beta}^{+}\right]=\operatorname{tr}\left[\tau_{i} \tau_{j}\right], & \mid i=1, \\
\operatorname{tr}\left[\tau_{i} \Lambda_{\beta}^{+} \tau_{j} \Lambda_{\beta}^{+}\right] \neq \operatorname{tr}\left[\tau_{i} \tau_{j}\right], & \mid i=2,3,4
\end{array}
$$

with $j$ being an integer and $j \in[1,4]$. This indicates that equations for $f_{1}(k ; P)$ with RML in the case of $\Lambda_{\beta}^{+}$are equivalent to those with $\mathrm{RL}$, whereas equations for $f_{2,3,4}(k ; P)$ are different from RL ones. Furthermore, if multiplying the Lorentz structure of the right-hand side of Eq. (11) in the case of $\Lambda^{-}$by $\tau_{i}$, one can get

$$
\begin{aligned}
\operatorname{tr}\left[\tau_{i} \Lambda^{-} \tau_{j} \Lambda^{-}\right] & =\operatorname{tr}\left[\tau_{i} \tau_{j}\right], \quad \mid i=1, \\
\Lambda^{-} \tau_{i} \Lambda^{-} & =\tau_{i}, \quad \mid i=2,3,4
\end{aligned}
$$

for any $j$. Thus, all equations in the case of $\Lambda^{-}$resemble those with RL. Therefore, we can even imagine before calculation that we will obtain eigenvalues and eigenvectors of the Bethe-Salpeter kernel in the case of $\Lambda^{-}$the same as their corresponding RL ones. This property makes us conclude that RML in the case of $\Lambda^{-}$for the pion is equivalent to RL. For this reason, the focus of this work is on RML in the case of $\Lambda_{\beta}^{+}$.

The procedure solving Eq. (11) is the common process finding eigenvalues and eigenvectors of the Bethe-Salpeter kernel [49]. After locating the pion mass, we simultaneously obtain the pion on-shell Bethe-Salpeter wave function, and it is the eigenvector associated with eigenvalue $\lambda\left(P^{2}=-M_{\pi_{i}}^{2}\right)=1$. The Bethe-Salpeter wave function can be normalized by the condition

$$
\left(\frac{\partial \ln (\lambda)}{\partial P^{2}}\right)^{-1}=\operatorname{tr} \int_{d q} \bar{\Gamma}(q ;-P) \chi(q ; P),
$$

with $\lambda$ the eigenvalue; $\bar{\Gamma}(q ; P)=\hat{C} \Gamma^{t}(-q ; P) \hat{C}^{-1}$ is the charge conjugation of Bethe-Salpeter amplitude $\Gamma(q ; P)=$ $S^{-1}\left(q_{+}\right) \chi(q ; P) S^{-1}\left(q_{-}\right)[50,51]$.

The normalized Bethe-Salpeter wave function can be used to define the pion decay constant, which is given by

$f_{\pi_{i}} P_{\mu}=Z_{2} \int_{d q} \operatorname{tr}\left[i \gamma_{5} \gamma_{\mu} S\left(q_{+}\right) \Lambda_{\beta} \Gamma(q ; P) \Lambda_{\beta} S\left(q_{-}\right)\right]$,

and the original pion decay constant definition with RL approximation can be obtained by letting $\Lambda_{\beta}$ approach $\mathbf{1}$.

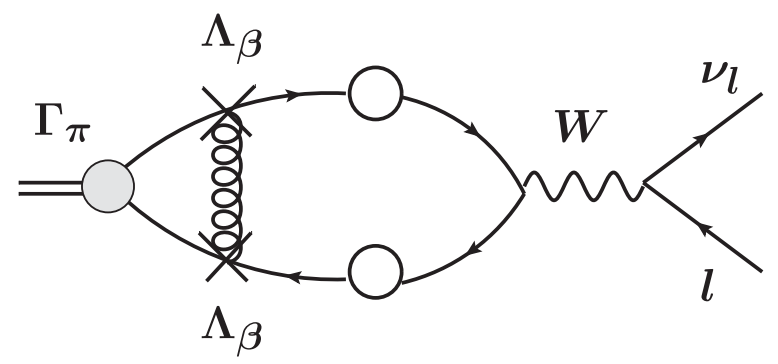

FIG. 2. Pion decay constant with modified-ladder approximation in Eq. (17). The filled circle represents the pion Bethe-Salpeter amplitude, and hollow circles are dressed quark propagators. The cross indicates to multiply a $\Lambda_{\beta}$ where it is marked.

A graphic representation of Eq. (17) is given in Fig. 2. It is pointed out as well in Ref. [52] that if loop momentum cutoff or nonlocal interactions are included, the pion decay constant must be modified accordingly, which is analogous with our case herein. Practically, if considering RML in the case of $\Lambda_{\beta}^{+}$, owing to the existing of relations in Eq. (14), the Lorentz structure of the pion decay constant on the right-hand side of Eq. (17) is distinct from the one with RL approximation. Additionally, the solution of the scalar function in the Bethe-Salpeter wave function that contributes the most to the decay constant, $f_{i}(k ; P) \mid i=2$, 3, with $\Lambda_{\beta}^{+}$is, in general, distinct from that with RL as well. Thus, combining these two effects, one cannot know in advance whether the pion decay constant with RML in the case of $\Lambda_{\beta}^{+}$remains the same as the one with RL, and it requires further consideration from numerical computation.

Given the normalized Bethe-Salpeter wave function, one can additionally consider the quantity associated with the quark condensate with RML approximation, which is of the form

$$
\rho_{\pi_{i}}=-Z_{4} \int_{d q} \operatorname{tr}\left[i \gamma_{5} S\left(q_{+}\right) \Lambda_{\beta} \Gamma(q ; P) \Lambda_{\beta} S\left(q_{-}\right)\right] .
$$

If considering RML in the case of $\Lambda_{\beta}^{+}$, it is noticed that, owing to the existing of relations in Eq. (14), the Lorentz structure of $\rho_{\pi_{i}}$ on the right-hand side of Eq. (18) is equivalent to the one with RL approximation, so that one can expect before any numerical computation that $\rho_{\pi_{i}}$ with RML in the case of $\Lambda_{\beta}^{+}$remains the same as the one with RL.

In particular, the preservation of the axial-vector Ward-Green-Takahashi identity in Eq. (6) yields the mass relation [11]

$$
f_{\pi_{i}} M_{\pi_{i}}^{2}=2 m^{\zeta} \rho_{\pi_{i}}(\zeta),
$$

which is known as the Gell-Mann-Oakes-Renner relation for the ground state pion [53]. Additionally, this mass relation entails that leptonic decay constants of pion radial 
excitations vanish in the chiral limit, and this is the consequence of chiral symmetry and its dynamical breaking in QCD [54]. It is necessary for any development on the scattering kernel to preserve this relation, and any breaking of this relation might indicate the breaking of the underlying symmetry. We may hope our RML in the case of $\Lambda_{\beta}^{+}$ would preserve this relation, and the justification will be discussed in the following section.

\section{NUMERICAL RESULTS}

Our work so far has consisted of setting up a general quark-antiquark scattering kernel in the Bethe-Salpeter equation, the modified-ladder approximation, especially in the case of $\Lambda_{\beta}^{+}$, derived directly from the vector and axial-vector Ward-Green-Takahashi identities. One of the dominant applications of the modified-ladder approximation is the pion, and we have specified the procedure calculating the pion mass, Bethe-Salpeter wave function, and their associated observables, i.e., decay constant, as well as the quantity associated with the quark condensate. In other words, we have designated the scattering kernel, which is one of the preknowledge inputs required in constructing the Bethe-Salpeter equation. The remaining unknown input is the gluon propagator. Once the scattering kernel and gluon propagator are both designated, the BetheSalpeter equation is well determined. Thus, in order to calculate pion properties, it now becomes necessary for us to implement the model of the gluon propagator. According to fruitful studies on this issue, we can apply the one introduced in Ref. [55], $D_{\mu \nu}(s)=\mathcal{P}_{\mu \nu} \mathcal{G}(s)$ :

$$
\mathcal{G}(s)=\frac{8 \pi^{2}}{\omega^{4}} D e^{-s / \omega^{2}}+\frac{8 \pi^{2} \gamma_{m} \mathcal{F}(s)}{\ln \left[\tau+\left(1+s / \Lambda_{\mathrm{QCD}}^{2}\right)^{2}\right]},
$$

where $\mathcal{P}_{\mu \nu}=\delta_{\mu \nu}-\frac{p_{\mu} p_{\nu}}{p^{2}} ; \gamma_{m}=12 /\left(33-2 N_{f}\right), \quad N_{f}=4$, $\Lambda_{\mathrm{OCD}}^{N_{f}=4}=0.234 \mathrm{GeV} ; \tau=e^{2}-1$; and $\mathcal{F}(s)=[1-\exp (-s /$ $\left.\left.\left[4 m_{t}^{2}\right]\right)\right] / s, m_{t}=0.5 \mathrm{GeV}$. The interaction in Eq. (20) involves a massive gluon scale on the domain at $s=0$, which is consistent with that determined in studies of QCD's gauge sector [56-58]. Parameters of interaction in Eq. (20) are taken as $D \omega=(0.82 \mathrm{GeV})^{3}$ and $\omega=0.5 \mathrm{GeV}$, which is the typical choice in a bulk of extant studies, and one can expect computed observables to be practically insensitive to the choice of $D$ or $\omega$ on a reasonable domain if $D \omega$ is kept stable [59].

Additionally, practical calculation must take a renormalization scale, at which physical quantities that we are interested are being considered. Of course, physical observables such as the pion mass and decay constant are independent of the chosen renormalization scale. We take the renormalization scale as $\zeta=0.3 \mathrm{GeV}$ herein, which is inspired by recent progress on the pion parton distribution function $[43,44]$, and the scale is originally from process-independent running coupling [60-63]. $\mathrm{RL}$ requires the renormalization-group-invariant light current-quark mass $\hat{m}=6.7 \mathrm{MeV}$, which corresponds to $m^{\zeta}=12.7 \mathrm{MeV}$. We take parameters with RML the same as those with RL, and, in this way, all the distinctions of pion properties caused by RML in the case of $\Lambda_{\beta}^{+}$compared to RL can be considered as induced by the variation of the scattering kernel.

\section{A. Masses of ground state $\pi_{0}$ and first radial excited state $\pi_{1}$}

After setting up computing inputs, let us consider our numerical results on the eigenvalue of the Bethe-Salpeter kernel, which corresponds to one at the physical point when the pion is on shell. The Nambu-Goldstone theorem predicts that the pion is massless in the chiral limit. To verify whether our modified kernel is well constructed, the first basic criteria we must consider is whether the NambuGoldstone theorem is manifest with RML in the case of $\Lambda_{\beta}^{+}$. We have numerically verified that $\Lambda_{\beta}^{+}$leads to a massless pion in the chiral limit; the RML in the case of $\Lambda_{\beta}^{+}$thus meets the first criterion, which is necessary to be manifest when developing a new scattering kernel.

Furthermore, let us consider the pion mass beyond the chiral limit. As we have seen in the previous section, eigenvalues of the Bethe-Salpeter kernel in homogeneous equations depend on the meson mass, and physical states are those corresponding to $\lambda=1$. The eigenvalue dependences on the meson mass of ground state $\pi_{0}$ and first radial excited state $\pi_{1}$ with RL and RML are illustrated in Fig. 3. We see that the eigenvalue dependence pattern on meson

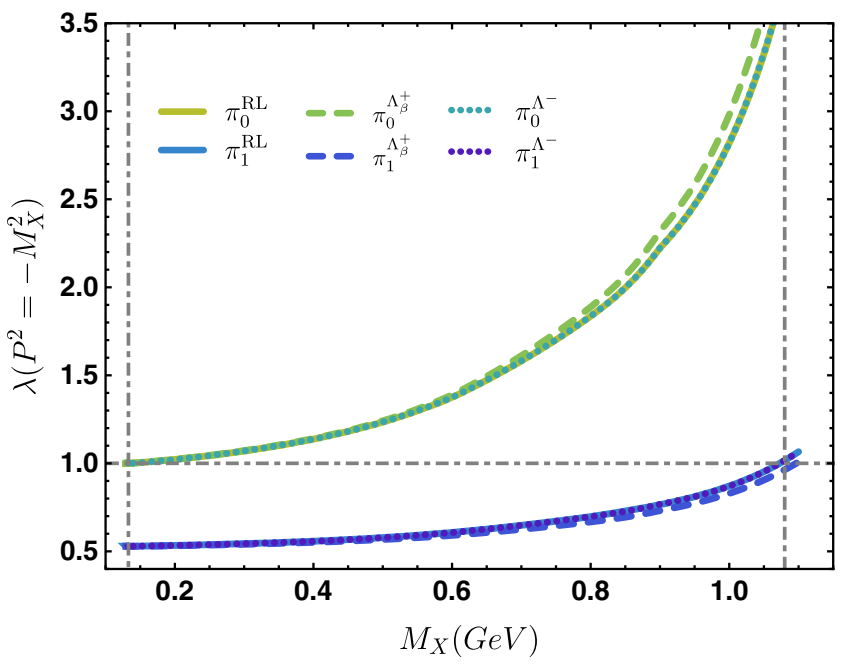

FIG. 3. Eigenvalue dependences on the meson mass of groundstate pion $\pi_{0}$ and first radial excited state $\pi_{1}$ with RL and RML $\left(\Lambda_{\beta}^{+}, \Lambda^{-}\right)$approximations. Curves: solid, RL; dashed, RML in the case of $\Lambda_{\beta}^{+}$; dotted, RML in the case of $\Lambda^{-}$. The vertical lines indicate the location where the curves cross $\lambda=1$, corresponding to the physical masses of these two states. 
mass is nearly identical among kernels. RML in the case of $\Lambda^{-}$gives exactly equivalent eigenvalue dependence with $\mathrm{RL}$, whereas $\Lambda_{\beta}^{+}$shows slightly distinction with them. This resembling pattern on eigenvalues of $\Lambda^{-}$can be easily understood, as we have pointed out earlier that all equations in this case are equivalent to those with RL; therefore, eigenvalues of the kernel will be the same as well. However, the nearly resembling pattern on eigenvalues with $\Lambda_{\beta}^{+}$is out of our expectation, since equations for $f_{2,3,4}(k ; P)$ are completely different between $\Lambda_{\beta}^{+}$and RL. Nevertheless, the eigenvalue behavior suggests that, despite scattering kernels in various approximations affecting the forms of the Bethe-Salpeter equation, physical masses of the ground state $\pi_{0}$ and first radial excited state $\pi_{1}$ are stable and nearly degenerate among different approximations

$M_{\pi_{0}}=0.133 \mathrm{GeV}, \quad M_{\pi_{1}}=1.08 \pm 0.03 \mathrm{GeV}$.

We include the variation for first radial excited state $\pi_{1}$ mass among kernels. Notably, the mass of $\pi_{1}$ herein is consistent with Ref. [54].

It is rather surprising to see nearly degenerate behavior in both $\pi_{0}$ and $\pi_{1}$ masses among various approximations in view of the fact that their Bethe-Salpeter equations with RML in the case of $\Lambda_{\beta}^{+}$and RL are, in general, not equivalent, there being apparent extra Dirac structures as a result of the inclusion of a multiplicative factor in the scattering kernel. The degenerate feature may be considered as the suggestion for a stable pion mass so long as the scattering kernel is constructed consistently. The qualification that has to be added here is the requirement that the kernel must be the solution of the symmetry-preserving vector and axial-vector Ward-Green-Takahashi identities.

\section{B. Bethe-Salpeter amplitude of ground state $\boldsymbol{\pi}_{\mathbf{0}}$}

Up to this point, we have seen results for eigenvalues of the Bethe-Salpeter kernel, which are associated with pion masses at a physical point; we now continue to consider eigenvectors, i.e., detailed structure of the Bethe-Salpeter amplitude. As an immediate example, we look attentively at the ground state $\pi_{0}$. Pion nonperturbative properties are all carried by its Bethe-Salpeter amplitude, so that the amplitude is fundamental itself for those attempting to describe the pion internal structure $[64,65]$. If we write the pion Bethe-Salpeter amplitude as $\Gamma(k ; P)=$ $\sum_{i=1}^{4} \tau_{i}(k ; P) F_{i}(k ; P)$, with $\tau_{i}(k ; P)$ given in Eq. (13), then $F_{i}(k, P)$ is the scalar function characterizing pion amplitude dependence on the relative and total momentum of dressed quark and dressed antiquark. Numerical results for the lowest-order Chebyshev projection of $F_{i}(k, P)$

$$
F_{i}\left(k^{2}\right):=\frac{2}{\pi} \int_{-1}^{1} d x \sqrt{1-x^{2}} U_{0}(x) F_{i}\left(k^{2}, x ; P^{2}\right),
$$
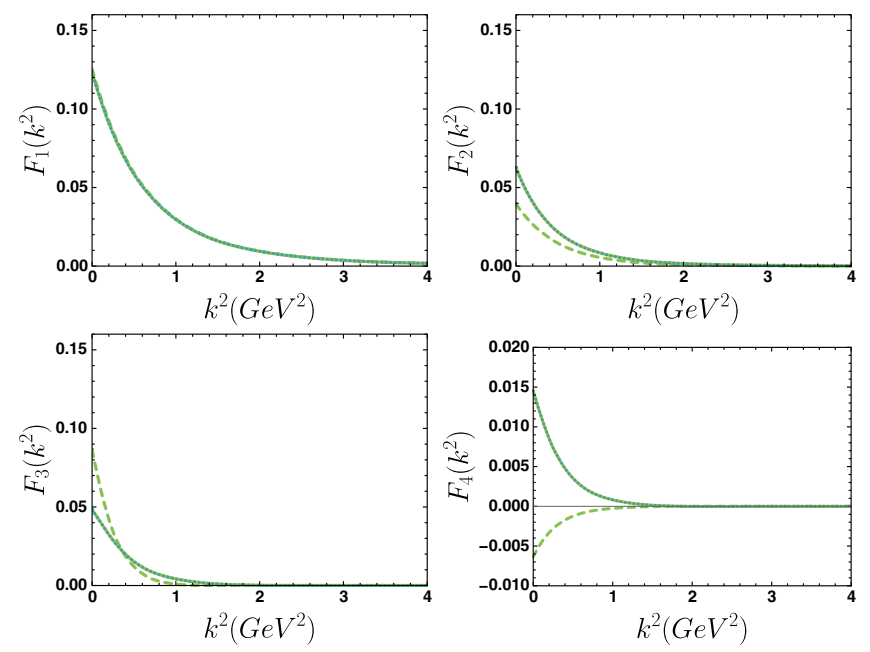

FIG. 4. Lowest-order Chebyshev projection of Bethe-Salpeter amplitudes $F_{1-4}\left(k^{2}\right)$ for ground-state pion $\pi_{0}$. In all panels, solid, RL; dashed, RML in the case of $\Lambda_{\beta}^{+}$; dotted, RML in the case of $\Lambda^{-}$. Row 1 , left, $F_{1}$; row 1 , right, $F_{2}$; row 2 , left, $F_{3}$; and row 2 , right, $F_{4}$.

for ground state $\pi_{0}$ with both $\mathrm{RL}$ and $\operatorname{RML}\left(\Lambda_{\beta}^{+}, \Lambda^{-}\right)$are shown in Fig. 4, where $k \cdot P=x \sqrt{k^{2} P^{2}}$ and $U_{0}(x)$ is the lowest-order Chebyshev polynomial of the second kind.

One apparent feature in all panels in Fig. 4 is the resembling pattern among all the lowest-order Chebyshev projection of Bethe-Salpeter amplitudes $F_{1-4}\left(k^{2}\right)$ with RML in the case of $\Lambda^{-}$(dotted curve) and those associated with the conventional RL (solid curve) approximation. According to the proof described above, all the Bethe-Salpeter equations are equivalent in these two cases; for this reason, we should obtain equivalent eigenvectors of the Bethe-Salpeter kernel. Numerical results herein have verified this statement. Therefore, we will focus on reviewing the difference on numerical results of Bethe-Salpeter amplitude between RML in the case of $\Lambda_{\beta}^{+}$and RL.

Unlike the nearly degenerate feature on pion masses as we described above, the lowest-order Chebyshev projection of the Bethe-Salpeter amplitude of $\pi_{0}$ expresses distinct behavior with RML in the case of $\Lambda_{\beta}^{+}$(dashed curve) and RL (solid curve). In detail, $F_{1}$ turns out to be equivalent in both cases, whereas $F_{2,3,4}$ behave differently with their dependence on relative momentum of the dressed quark and dressed antiquark in the infrared region. If we consider $\mathrm{RML}$ in the case of $\Lambda_{\beta}^{+}$, the general decreasing behavior of $F_{2}$ remains, whereas $F_{2}\left(k^{2}=0\right)$ is relatively smaller in comparison to that with RL. $F_{3}$ keeps the deceasing behavior as well, and $F_{3}(0)$ is relatively larger. $F_{4}$ turns out to be negative.

One might find explanations for these features from equations we have mentioned above. Equations for $F_{1}$ with RML in the case of $\Lambda_{\beta}^{+}$are equivalent to those with RL, whereas equations for $F_{2,3,4}$ with $\Lambda_{\beta}^{+}$differ from the ones 
with RL [see Eq. (14)]. Consequently, it may be possible that these two approximations yield the same $F_{1}$ yet different $F_{2,3,4}$. The appearance of different $F_{2,3,4}$ can be employed to analyze the content of the Bethe-Salpeter wave function with a specific orbital angular momentum [66]. These features in the Bethe-Salpeter amplitude indicate that in these two approximations it is possible to have various s- and p-wave contributions; meson structure functions will thus have more diverse properties rather than the unique behavior.

\section{Decay constant of ground state $\pi_{0}$ and the GMOR relation}

The distinction in ground state $\pi_{0}$ Bethe-Salpeter amplitude with RML in the case of $\Lambda_{\beta}^{+}$and RL may lead to a distinct decay constant as defined in Eq. (17). However, in practice, we find

$$
f_{\pi_{0}}^{\mathrm{RL}}=92.3 \mathrm{MeV}, \quad f_{\pi_{0}}^{\Lambda_{\beta}^{+}}=93.5 \mathrm{MeV},
$$

in comparison to that in experiment $f_{\pi_{0}}^{\exp }=91.9 \pm$ $3.54 \mathrm{MeV}$ [67]. Therefore, one may notice that both $f_{\pi_{0}}^{\mathrm{RL}}$ and $f_{\pi_{0}}^{\Lambda_{\beta}^{+}}$are compatible with the experimental value by relative errors within 2\%. Again, it is rather surprising to see a nearly degenerate pion decay constant among various approximations in view of the fact that their Bethe-Salpeter amplitudes are, in general, not equivalent (see Fig. 4). The degenerate feature may also be considered as the suggestion for a stable pion decay constant so long as the scattering kernel is constructed from Ward identities. The pion decay constant together with pion mass is a physical observable, which should be, in principle, invariant among models, and our practical calculation shows this is the case herein. Other physical observables, such as the pion parton distribution function, we can expect will also be invariant with any symmetry-preserving modification of the Bethe-Salpeter scattering kernel, and this remains to be justified by future studies.

In order to examine whether the preservation of the GMOR relation still holds with RML in the case of $\Lambda_{\beta}^{+}$and RL approximations, we include the calculation the quantity associated with the quark condensate defined in Eq. (18) and obtain

$$
\rho_{\pi_{0}}^{\mathrm{RL}}(\zeta)=(0.252 \mathrm{GeV})^{2}, \quad \rho_{\pi_{0}}^{\Lambda_{\beta}^{+}}(\zeta)=(0.250 \mathrm{GeV})^{2} .
$$

We have seen in the preceding section that $\rho_{\pi_{0}}$ with $\Lambda_{\beta}^{+}$is equivalent to that with RL based on analytical analysis, and practical numerical results turn out to be consistent with this statement. Now we can consider the maintenance of the
GMOR relation in Eq. (19). If it is maintained, then the ratio of decay constant $f_{\pi_{0}}$ and $\rho_{\pi_{0}}$ is

$$
f_{\pi_{0}} / \rho_{\pi_{0}}(\zeta)=2 m^{\zeta} / M_{\pi_{0}}^{2} .
$$

In practice, we use the same light current quark mass $m^{\zeta}=12.7 \mathrm{MeV}$ with RML in the case of $\Lambda_{\beta}^{+}$and with RL, and we additionally notice that ground state $\pi_{0}$ mass degenerate $M_{\pi_{0}}=0.133 \mathrm{GeV}$ in these two approximations; therefore, the ratio on the right-hand side of Eq. (25) with $\Lambda_{\beta}^{+}$is exactly equivalent to that with RL, viz., $2 m^{\zeta} / M_{\pi_{0}}^{2}=1.436 \mathrm{GeV}^{-1}$. When considering the lefthand side of Eq. (25) with these two approximations, we find $f_{\pi_{0}}^{\mathrm{RL}} / \rho_{\pi_{0}}^{\mathrm{RL}}(\zeta)=1.451 \mathrm{GeV}^{-1}$ and $f_{\pi_{0}^{\prime}}^{\Lambda_{\beta}^{+}} / \rho_{\pi_{0}^{+}}^{\Lambda_{\beta}^{+}}(\zeta)=$ $1.496 \mathrm{GeV}^{-1}$; hence, the GMOR relation is preserved with RL and RML by a relative error within 5\%.

Notably, considering RML in the case of $\Lambda_{\beta}^{+}$, the GMOR relation is preserved only if we follow the definition of pion decay constant in Eq. (17) with a graphic representation given in Fig. 2 and the definition of $\rho_{\pi_{0}}$ in Eq. (18), in which the multiplicative factor is included. This might suggest that, when considering physical observables making use of the Bethe-Salpeter amplitude with the modified-ladder approximation, one must take into account the possible modification on the expression associated with physical observables of interest.

Additionally, given that the GMOR relation connects the current quark mass, pion mass, decay constant, and the quantity associated with the quark condensate, one can start from it and obtain the above quantities from one to another. However, if a theory can give these quantities simultaneously, it would be not easy for them to satisfy the GMOR relation with a high accuracy. Therefore, besides the Nambu-Goldstone theorem, the GMOR relation can serve as a second criterion, which is necessary to manifest for the pion when developing a new scattering kernel.

\section{CONCLUSION}

In this work, we explored the possible rainbow modifiedladder approximation, derived directly from the vector and axial-vector Ward-Green-Takahashi identities. Starting from Ward identities in rainbow approximation, we obtain two equations for the quark-antiquark scattering kernel in the Bethe-Salpeter equation, Eqs. (5) and (7). Then the quark-antiquark scattering kernel is assumed to include a multiplicative factor in comparison to that with the conventional rainbow ladder approximation. Apart from 1, two nontrivial solutions for the multiplicative factor are found, as outlined in Eq. (10), corresponding to the rainbow modified-ladder approximation. There is a distinction with the modified-ladder approximation scattering kernel in comparison to that in the ladder approximation, which is that it owns momentum dependence described by the quark 
momentum and/or the vector part of the quark propagator. In consequence of this, it may lead to some impacts on properties of the system that one is interested in.

As an application of this rainbow modified-ladder approximation, we study the pion. We have first numerically verified that the Nambu-Goldstone theorem is manifest with the modified-ladder approximation. Then the most remarkable result is that the pion masses of both ground state $\pi_{0}$ and first radial excited state $\pi_{1}$ are degenerate with rainbow ladder and rainbow modified-ladder approximations, as illustrated in Fig. 3. The degenerate feature may suggest a stable pion mass so long as the scattering kernel is constructed from the symmetry-preserving Ward identities. The Bethe-Salpeter amplitude of the ground state $\pi_{0}$ is then considered. Unlike the degenerate feature in pion masses, the Bethe-Salpeter amplitude of $\pi_{0}$ expresses distinct behavior with RML in the case of $\Lambda_{\beta}^{+}$and RL, as given

[1] M. Gell-Mann, Phys. Lett. 8, 214 (1964).

[2] G. Zweig, CERN Report No. CERN-TH-401, 1964, https:// cds.cern.ch/record/352337; Report No. CERN-TH-412, 1964, https://cds.cern.ch/record/570209.

[3] R. Feynman, Conf. Proc. C690905, 237 (1969), https:// inspirehep.net/literature/57407.

[4] J. Bjorken and E. A. Paschos, Phys. Rev. 185, 1975 (1969).

[5] S. Aoki et al. (Flavour Lattice Averaging Group), Eur. Phys. J. C 80, 113 (2020).

[6] C. D. Roberts and A. G. Williams, Prog. Part. Nucl. Phys. 33, 477 (1994).

[7] P. Maris and C. D. Roberts, Int. J. Mod. Phys. E 12, 297 (2003).

[8] G. Eichmann, H. Sanchis-Alepuz, R. Williams, R. Alkofer, and C. S. Fischer, Prog. Part. Nucl. Phys. 91, 1 (2016).

[9] J. M. Pawlowski, Ann. Phys. (Amsterdam) 322, 2831 (2007).

[10] A. K. Cyrol, M. Mitter, J. M. Pawlowski, and N. Strodthoff, Phys. Rev. D 97, 054006 (2018).

[11] P. Maris, C. D. Roberts, and P. C. Tandy, Phys. Lett. B 420, 267 (1998).

[12] Y.-B. Dai, C.-S. Huang, and D.-S. Liu, Phys. Rev. D 43, 1717 (1991).

[13] T. Maskawa and H. Nakajima, Prog. Theor. Phys. 52, 1326 (1974).

[14] T. Maskawa and H. Nakajima, Prog. Theor. Phys. 54, 860 (1975).

[15] E. Salpeter and H. Bethe, Phys. Rev. 84, 1232 (1951).

[16] S. Mandelstam, Phys. Rev. D 20, 3223 (1979).

[17] P. Watson, W. Cassing, and P. Tandy, Few Body Syst. 35, 129 (2004).

[18] H. H. Matevosyan, A. W. Thomas, and P. C. Tandy, Phys. Rev. C 75, 045201 (2007).

[19] C. S. Fischer, D. Nickel, and J. Wambach, Phys. Rev. D 76, 094009 (2007). in Fig. 4. It is then noticed that the distinction on $\pi_{0}$ BetheSalpeter amplitude does not affect the pion decay constant, in the case RML, and it is generally equivalent to the one with RL. Consequently, the GMOR relation is also numerically verified to be preserved with the modified-ladder approximation.

The complexity of numerical computation with rainbow modified-ladder approximation does not increase dramatically compared to rainbow ladder approximation, so that the study herein can be easily extended to further investigation on other mesons such as heavy quarkonium and mesons with nonzero spin.

\section{ACKNOWLEDGMENTS}

We acknowledge valuable input from Daniele Binosi, Muyang Chen, Fei Gao, and Craig Roberts.
[20] C. S. Fischer and R. Williams, Phys. Rev. D 78, 074006 (2008).

[21] W. Heupel, T. Goecke, and C. S. Fischer, Eur. Phys. J. A 50, 85 (2014).

[22] S.-x. Qin, EPJ Web Conf. 113, 05024 (2016).

[23] S.-x. Qin, Few Body Syst. 57, 1059 (2016).

[24] D. Binosi, L. Chang, J. Papavassiliou, S.-X. Qin, and C. D. Roberts, Phys. Rev. D 93, 096010 (2016).

[25] A. Bender, C. D. Roberts, and L. Von Smekal, Phys. Lett. B 380, 7 (1996).

[26] L. Chang and C. D. Roberts, Phys. Rev. Lett. 103, 081601 (2009).

[27] R. Williams, EPJ Web Conf. 3, 03005 (2010).

[28] S.-x. Qin and C. D. Roberts, arXiv:2009.13637.

[29] P. Qin, S.-x. Qin, and Y.-x. Liu, Phys. Rev. D 101, 114014 (2020).

[30] L. Chang, I. Cloet, J. Cobos-Martinez, C. Roberts, S. Schmidt, and P. Tandy, Phys. Rev. Lett. 110, 132001 (2013).

[31] R. Williams, Phys. Lett. B 798, 134943 (2019).

[32] G. Eichmann, C. S. Fischer, W. Heupel, N. Santowsky, and P. C. Wallbott, Few Body Syst. 61, 38 (2020).

[33] M. Chen and L. Chang, Chin. Phys. C 43, 114103 (2019).

[34] L. Chang, M. Chen, and Y.-X. Liu, Phys. Rev. D 102, 074010 (2020).

[35] J. C. Ward, Phys. Rev. 78, 182 (1950).

[36] H. Green, Proc. Phys. Soc. London Sect. A 66, 873 (1953).

[37] Y. Takahashi, Nuovo Cimento 6, 371 (1957).

[38] J. C. Bloch, Phys. Rev. D 66, 034032 (2002).

[39] G. Eichmann, C. S. Fischer, E. Weil, and R. Williams, Phys. Lett. B 797, 134855 (2019); 799, 135029 (2019).

[40] G. Eichmann, C. S. Fischer, and R. Williams, Phys. Rev. D 101, 054015 (2020).

[41] L. Gutiérrez-Guerrero, A. Bashir, M. A. Bedolla, and E. Santopinto, Phys. Rev. D 100, 114032 (2019). 
[42] T. Frederico, D. C. Duarte, W. de Paula, E. Ydrefors, S. Jia, and P. Maris, arXiv:1905.00703.

[43] M. Ding, K. Raya, D. Binosi, L. Chang, C. D. Roberts, and S. M. Schmidt, Phys. Rev. D 101, 054014 (2020).

[44] M. Ding, K. Raya, D. Binosi, L. Chang, C. D. Roberts, and S. M. Schmidt, Chin. Phys. C 44, 031002 (2020).

[45] F. Gao and M. Ding, Eur. Phys. J. C 80, 1171 (2020).

[46] M. Barabanov et al., Prog. Part. Nucl. Phys. 116, 103835 (2021).

[47] A. C. Aguilar et al., Eur. Phys. J. A 55, 190 (2019).

[48] G. Arfken, Mathematical Methods for Physicists (Academic Press, New York, 1985).

[49] H. Sanchis-Alepuz and R. Williams, Comput. Phys. Commun. 232, 1 (2018).

[50] N. Nakanishi, Phys. Rev. 138, B1182 (1965).

[51] N. Nakanishi, Phys. Rev. 139, B1401 (1965).

[52] K. Naito, K. Yoshida, Y. Nemoto, M. Oka, and M. Takizawa, Phys. Rev. C 59, 1095 (1999).

[53] M. Gell-Mann, R. Oakes, and B. Renner, Phys. Rev. 175, 2195 (1968).

[54] A. Holl, A. Krassnigg, and C. Roberts, Phys. Rev. C 70, 042203 (2004).

[55] S.-x. Qin, L. Chang, Y.-x. Liu, C. D. Roberts, and D. J. Wilson, Phys. Rev. C 84, 042202 (2011).
[56] A. Aguilar, D. Binosi, and J. Papavassiliou, J. High Energy Phys. 07 (2010) 002.

[57] D. Binosi, D. Ibanez, and J. Papavassiliou, Phys. Rev. D 86, 085033 (2012).

[58] D. Binosi and R.-A. Tripolt, Phys. Lett. B 801, 135171 (2020).

[59] M. Chen, M. Ding, L. Chang, and C. D. Roberts, Phys. Rev. D 98, 091505 (2018).

[60] D. Binosi, C. Mezrag, J. Papavassiliou, C. D. Roberts, and J. Rodriguez-Quintero, Phys. Rev. D 96, 054026 (2017).

[61] J. Rodríguez-Quintero, D. Binosi, C. Mezrag, J. Papavassiliou, and C. D. Roberts, Few Body Syst. 59, 121 (2018).

[62] C. D. Roberts and S. M. Schmidt, Eur. Phys. J. Special Topics 229, 3319 (2020).

[63] C. D. Roberts, Symmetry 12, 1468 (2020).

[64] P. Maris and C.D. Roberts, Phys. Rev. C 56, 3369 (1997).

[65] S.-x. Qin, L. Chang, Y.-x. Liu, C. D. Roberts, and D. J. Wilson, Phys. Rev. C 85, 035202 (2012).

[66] T. Hilger, M. Gomez-Rocha, and A. Krassnigg, Eur. Phys. J. C 77, 625 (2017).

[67] P. Zyla et al. (Particle Data Group), Prog. Theor. Exp. Phys. 2020, 083C01 (2020). 